Oper Orthop Traumatol 2015 $\cdot 27: 4$

DOI 10.1007/s00064-014-0359-3

๑) Springer-Verlag Berlin Heidelberg 2015

\section{Schriftleitung Operative Orthopädie und Traumatologie}

\section{Die Zeitschrift Operative Orthopädie und Traumatologie dankt den Gutachtern 2014}

\begin{abstract}
Die Schriftleitung der Zeitschrift Operative Orthopädie und Traumatologie dankt den Herausgebern der Zeitschrift und insbesondere allen weiteren Experten für ihre Unterstützung als wissenschaftliche Gutachter im Jahr 2014. Durch ihren Einsatz garantieren sie im Rahmen des Peer-Review-Verfahrens der eingereichten Manuskripte die hohe Qualität und klinische Relevanz der Zeitschrift Operative Orthopädie und Traumatologie.
\end{abstract}

\section{Dr. Ralph Akoto}

Chirurgisch-Traumatologisches

Zentrum, Asklepios Klinik

St. Georg, Hamburg

PD Dr. Michael Amlang

Universitätsklinikum Carl Gustav Carus, Technische Universität Dresden

PD Dr. Hermann Anetzberger Orthopädische Praxis, München

Dr. Jörg Arnholdt

Orthopädische Klinik, König-

Ludwig-Haus Würzburg

\section{Dr. Rohit Arora}

Universitätsklinik für

Unfallchirurgie, Innsbruck

PD Dr. Peter Balcarek

Klinik für Unfallchirurgie,

Universitätsmedizin Göttingen

Prof. Dr. Roland Becker

Abteilung Orthopädie, Städtisches

Klinikum Brandenburg

Dr. Knut Beitzel

Klinikum rechts der Isar, TU München

Dr. Berthold Bickert

Berufsgenossenschaftliche

Unfallklinik, Ludwigshafen
PD Dr. Sepp Braun

Klinikum rechts der Isar, TU München

PD Dr. Peter Brucker

Klinikum rechts der Isar, TU München

Prof. Dr. Ulf Culemann

Allgemeines Krankenhaus

Unfallchirurgie, Celle

PD Dr. Michael Dienst

OCM Orthopädische Chirurgie München

Dr. Florian Dirisamer

Orthopädie \& Sportchirurgie, Puchenau

Dr. Jörn Dohle

OGAM - Orthopädie, Wuppertal

PD Dr. Hans Josef Erli

Vivantes Humboldt-Klinikum Klinikf.

Unfallchirurgie und Orthopädie, Berlin

PD Dr. Norman Espinosa

Uniklinik Balgrist, Zürich

Prof. Dr. Karl-Heinz Frosch

Chirurgisch-Traumatologisches Zentrum, Asklepios Klinik St. Georg, Hamburg

Prof. Dr. Florian Gebhard Klinik für Unfallchirurgie, Ulm

Dr. Jens Geerling

Diakoniekrankenhaus

Friederikenstift, Hannover

Dr. Christian Gerhardt

Centrum für Muskuloskeletale Chirurgie, Charité -Universitätsmedizin Berlin

Prof. Dr. Peter Hahn

Handchirurgie, Vulpius

Klinik, Bad Rappenau

Dr. Mirco Herbort

Universitätsklinikum Münster

PD Dr. Maik Hoberg

Orthopädische Klinik, König-

Ludwig-Haus Würzburg

PD Dr. Bernd Hohendorff

Klinik für Unfallchirurgie und

Orthopädie, Elbe Kliniken Stade

Dr. Boris Holzapfel

Orthopädische Klinik, König-

Ludwig-Haus Würzburg

PD Dr. Chlodwig Kirchhoff

Klinikum rechts der Isar, TU München
Dr. Stefan Kirschner

Klinik und Poliklinik für Orthopädie,

Universitätsklinikum Dresden

Prof. Dr. Michael Klinger

Abteilung Orthopädie,

Universitätsmedizin Göttingen

Dr. Hendrik Kohlhof

Orthopädisch-Unfallchirurgische

Poliklinik, Universitätsklinikum Bonn

Prof. Dr. Djordje Lazovic

Pius-Hospital, Oldenburg

PD. Dr. Andreas Lenich

Klinikum rechts der Isar, München

PD Dr. Kai Megerle

Klinikum rechts der Isar, München

Dr. Sven Ostermeier

Praxis und Gelenk-Klinik, Gundelfingen

Dr. Peter Pennekamp

Orthopädisch-Unfallchirurgische

Poliklinik, Universitätsklinikum Bonn

Dr. Thomas Pillukat

Rhön-Klinikum AG Klinik für

Handchirurgie, Bad Neustadt a.d. Salle

Dr. Jörg Rether

Berufsgenossenschaftliche

Unfallklinik, Tübingen

Dr. Falk Reuther

DRK Kliniken Berlin Koepenick

Dr. Martin Richter

Klinik für Handchirurgie,

Malteser Krankenhaus Seliger

Gerhard Bonn/Rhein-Sieg

Prof. Dr. Peter Schandlmaier

DONAUISAR Klinikum

Deggendorf und Landau

Dr. Christian Spies

Abteilung für Hnadchirurgie,

Vulpius Klinik, Bad Rappenau

Prof. Dr. Jörn Steinbeck

Orthopädische Praxis/

Praxisklinik, Münster

Prof. Dr. André Steinert

Orthopädische Klinik, König-

Ludwig-Haus Würzburg
Prof. Dr. Ulrich Stöckle

Berufsgenossenschaftliche

Unfallklinik, Tübingen

PD Dr. Mark Tauber ATOS Klinik München

Dr. Katja Tecklenburg

medalp Imst - Zentrum für ambulante Chirurgie Betriebs $\mathrm{GmbH}$, Imst

Prof. Dr. Markus Tingart Klinik für Orthopädie und Unfallchirurgie Universitätsklinikum Aachen

Prof. Dr. Thomas Tischer Orthopädische Klinik und Poliklinik, Universitätsmedizin Rostock

Prof. Dr. Rüdiger von

Eisenhart-Rothe

Klinikum rechts der Isar, TU München

Dr. Markus Wambacher

Universitätsklinikum für Unfallchirurgie, Innsbruck

Prof. Dr. Johannes Zeichen

Klinik für Unfallchirurgie und Orthopädie, Johannes Wesling Klinikum Minden 